\title{
Rare earth-doped integrated glass components: modeling and optimization
}

\author{
Lumholt, Ole; Bjarklev, Anders Overgaard; Rasmussen, Thomas; Lester, Christian
}

Published in:

Journal of Lightwave Technology

Link to article, DOI:

$10.1109 / 50.365216$

Publication date:

1995

Document Version

Publisher's PDF, also known as Version of record

Link back to DTU Orbit

Citation (APA):

Lumholt, O., Bjarklev, A. O., Rasmussen, T., \& Lester, C. (1995). Rare earth-doped integrated glass components: modeling and optimization. Journal of Lightwave Technology, 13(2), 275-282.

https://doi.org/10.1109/50.365216

\section{General rights}

Copyright and moral rights for the publications made accessible in the public portal are retained by the authors and/or other copyright owners and it is a condition of accessing publications that users recognise and abide by the legal requirements associated with these rights.

- Users may download and print one copy of any publication from the public portal for the purpose of private study or research.

- You may not further distribute the material or use it for any profit-making activity or commercial gain

- You may freely distribute the URL identifying the publication in the public portal

If you believe that this document breaches copyright please contact us providing details, and we will remove access to the work immediately and investigate your claim. 


\title{
Rare Earth-Doped Integrated Glass Components: Modeling and Optimization
}

\author{
Ole Lumholt, Anders Bjarklev, Member, IEEE, Thomas Rasmussen, and Christian Lester
}

\begin{abstract}
For the integrated optic erbium-doped phosphate silica amplifier, a comprehensive model is presented which includes high-concentration dissipative ion-ion interactions. Based on actual waveguide parameters, the model is seen to reproduce measured gains closely. A rigorous design optimization is performed, and the influence of variations in the launched pump power, the core cross section, the waveguide length, the erbium concentration, and the background losses are evaluated. Optimal design proposals are given, and the process reproducibility of the proposed optimal design is examined. Requirements for process parameter control in the wafer fabrication are set up.
\end{abstract}

\section{INTRODUCTION}

$\mathbf{F}$ EW components have revolutionized the area of optical research as the rare earth-doped optical amplifier and laser. This is mainly due to the eye safe transition around $1.55 \mu \mathrm{m}$ of erbium and the $1.3 \mu \mathrm{m}$ emission of neodymium or praseodymium which, respectively, coinside with the low-loss window and the zero dispersion wavelength of standard optical fibers. Although fiber-versions of these components are already commercially available, the integrated optic version seems to be competitive and in some ways unsurpassed by fibers. This is because integrated optics offer prospects of active components to be integrated on the same substrate as filters, couplers, taps, and multiplexers so optical switching and multiplexing can be made locally on the wafer. The integration offers robust devices, compact size, multiple device fabrication on the same substrate, and packaging at lower cost.

From the variety of rare earth ions and integrated optic substrate materials, the main part of this paper will focus on the erbium-doped glass components, although differences from other rare earth ions are discussed when significant. Glass is chosen as host, as it is stable to changes of environment, has a low propagation loss, and the potential of low-loss coupling to standard optical fibers. The $1.55 \mu \mathrm{m}$ narrow linewidth erbium source is of the utmost importance for high-bit-rate long-distance fiber optic communication and for coherent optical transmission, but also for spectroscopic, telemetry [1], and special metrological applications [2]. Combined with the advantages of integration and dense packing, a miniaturized diode-pumped eye safe source will be extremely attractive for the medical industry and applications as sensors, laser

Manuscript received October 7, 1993; revised October 27, 1994. This work was supported by the Danish Technical Research Council and by the Agency of Industry and Trade, Denmark.

The authors are with the Center for Broadband Telecommunications, Electromagnetics Institute, Technical University of Denmark, DK-2800 Lyngby, Denmark.

IEEE Log Number 9407810. radars, free-space communication, tactical training range finding, security systems [3], and as superradiant sources for optical gyroscopes [4]. In addition, other wavelengths could be reached by using the transitions ranging from the IR part of the spectrum to the visible part, which are accessible through upconversion pumping schemes. In particular, compact visible laser sources are needed for optical disk storage purposes [5], medical, and sensor applications.

Accurate numerical modeling of rare earth-doped integrated optic componentsare of extreme importance in the development and processing of high-performance devices, and are required far more than for fiber optimization. Compared to active fibers, we have, after the fabrication, one adjustment parameter less to compensate for minor deviations during processing, as the doped waveguide length is determined during the fabrication. This leaves the pump power as the only adjustable design parameter, and sets up requirements for accurate predicted design proposals before processing. In this paper, we present an overview of different rare earth incorporation techniques of active wafers, with their limitations to certain glass compositions, as the host glass development can be viewed as part of the integrated optical amplifier optimization. An overview of applications and the state of the art is given. For illustration and simplification, we then concentrate on the erbium-doped phosphor/silica-core silicaon-silicon wafer amplifier, present a numerical model for this component, verify the model with measurements, and perform a rigorous design optimization of the high-gain component.

\section{HOW TO INCORPORATE RARE-EARTH IONS IN GLASS}

Diffusion and deposition are two basic different methods used for core layerintroduction in integrated optics (IO). Channel waveguides are formed in the former by diffusing index raising materials through a slide in a substrate surface covering mask, while for deposition, photolithography can be used to write structures on the surface, whereafter reactive ion-etching (RIE) is used to remove any core areas not masked. Buried waveguides are made in the diffusion process by extraction of core material near the surface [6], while in the deposition, an extra cladding layer is deposited on top of the ridged waveguide. Also, the techniques by which rare earth (RE) ions are impregnated to the core most often differ from these processes. Generally, rare earth ions are introduced into the core glass of deposited waveguides, while the core is introduced into the rare earth glass for diffused components. Fig. 1 shows different combinations of core layer fabrication 


\begin{tabular}{|c|c|c|}
\hline Core layer fabrication: & Rare earth incorp & \\
\hline HIPOX & Ion implantation & A \\
\hline LPCVD & Ion implantation & B \\
\hline PECVD & Chelates & c \\
\hline FHD & $\begin{array}{l}\text { Solution } \\
\text { Aerosol } \\
\text { Chelates }\end{array}$ & $\begin{array}{l}D \\
E \\
F\end{array}$ \\
\hline Sputter & $\begin{array}{l}\text { RF sputter } \\
\text { lon beam } \\
\text { lon implantation }\end{array}$ & $\begin{array}{l}\mathrm{G} \\
\mathrm{H} \\
\mathrm{I}\end{array}$ \\
\hline Ion exchange & $\begin{array}{l}\text { Bulk } \\
\text { lon implantation } \\
\text { Erbium } \\
\text { Composite }\end{array}$ & $\begin{array}{l}J \\
K \\
L \\
M\end{array}$ \\
\hline
\end{tabular}

Fig. 1. Combinations of core layer fabrication and rare earth incorporation techniques. HIPOX: high-pressure steam oxidization. LPCVD: low-pressure chemical vapor deposition. PECVD: plasma-enhanced CVD. FHD: flame hydrolysis deposition. A, B: [10], C: [11], D: [12], E: [13], F: [14], G: [15], H: [16], I: [17], J: [18], K: [19], L: [20], M: [21].

techniques and rare earth incorporation processes that have been published within the last few years.

One should bear in mind that phosphate glasses (or, alternatively,alumino-silicate glasses) seem to be the best suited host materials for most applications of rare earth-doped IO amplifiers. This is because phosphate (and aluminum) act as network modifiers in the glass, thereby eliminating the clustering tendency as reported for highly RE-doped fibers [7]. Phosphate increases the refractive index, and is therefore often used as a core material in deposited waveguides. In addition, it has a lack, compared to Ge-silica, of the unwanted excited state absorption, ESA, around the $1.35 \mu \mathrm{m}$ $\mathrm{Nd}$ transition [8]. However, as phosphate glasses have large effective phonon frequencies (high nonradiative transition rates), slightly reduced quantum efficiencies can be expected for some compositions [9].

\section{MOdELING}

The modeling of rare earth-doped channel waveguides differs on severalpoints from the modeling of rare earth-doped fibers. One difference is the noncircular symmetric core cross section, which typically are rectangular for deposited and elliptical for diffused waveguides. Another is the noncircular symmetric rare earth doping profile; this can be a rectangular step-like profile for while deposition or solution doping, vertically Gaussian shaped for doping by ion implantation, homogeneous with the core confined within the doped area for ion-exchanged waveguides, or located outside the core as in the case for the composite waveguides.

Background losses of buried channel waveguides are around five-six decades larger than in fibers, typically between a few tenths to several $\mathrm{dB} / \mathrm{cm}$. Local deformations at material surfaces during manufacturing have a very significant impact upon the integrated component, while in fiber preforms, they are stretched and smoothed out on several thousand times longer lengths during the drawing. The background loss results from a combination of internal scattering, surface scattering, sidewall scattering (for rectangular cores), and Rayleigh scattering due to the high RE concentration. The latter is the most insignificant of these, i.e., a high-concentration ( 86 . $10^{24}$ ions $/ \mathrm{cm}^{3}$ ) Er fiber only exhibited a minor loss increase [22], while the sidewall scattering is the most difficult to handle, especially for small channel widths. The main contribution to this is the mask and etching process which creates a kind of waved walls [23]. Surface scattering is due to imperfections in the core-cladding boundaries and depends on the glass film processing, while the internal scattering depends on the purity of the deposited glass. Buried planar channel waveguides have been made with losses of $0.026 \mathrm{~dB} / \mathrm{cm}$ [24], although typical values are three-ten times higher. Finally, rare earth concentrations two orders of magnitude higher than in fibers are necessary to achieve short lengths of amplifiers. At these concentration levels, ion-ion interactions, such as excited ion migration and cooperative upconversion or cross relaxation, are strongly present, yielding decreased amplifier performance. On the other hand, the fiber problem with RE clusters is not considered as a problem for RE-doped integrated components with an ample amount of phosphor or aluminum in the core.

Models that take some or all of the above-mentioned aspects into account have been presented [25]-[31], but with quite different solutions to the problems. $A b$ initio calculations of a multilevel system are extremely complicated, so one has to weigh the modeling between, on the one hand, the full scale model with all dissipative processes included and, on the other hand, a quick useful design tool. For erbium systems, the major cause for inefficiency is the cooperative upconversion, which schematically is shown on the left side of Fig. 2 . Two excited Er ions can transfer energy from one to the other, leaving the first in the ground state and the second in the ${ }^{4} I_{9 / 2}$ state with a quick nonradiative relaxation back to ${ }^{4} I_{13 / 2}$. The net result is a conversion of pump photons into heat. A simple approximation to this process is made by including a term proportional to $k_{2} n_{2}^{2}$ in the rate equations. $n_{2}$ is the population in the upper laser level, and $k_{2}$ is the upconversion constant of about $1 \cdot 10^{-23} \mathrm{~m}^{3} / \mathrm{s}$ [32]. Another model also includes higher order upconversion processes [29], while others have found that the upconversion constant is concentration-dependent [31], yielding a cubic equation for the determination of the population inversion. A similar equation appears when the performance degradation is modeled through the observed relation between fluorescence lifetime and the concentration [28], as will be described in detail below. The neodymium system acts different. Due to intermediate states between the upper and lower laser level, the inefficiency is caused by cross relaxations to these, involving one excited ion and one in the ground state, followed by a quick relaxation to the ground state (illustrated on the right hand side of Fig. 2). A term proportional to $k_{i j} n_{i} n_{j}$ should be applied for the simple approximation in this case, $k_{i j}$ being the cross-relaxation coefficient between levels $i$ and $j$.

For illustration, we have concentration our modeling on erbium-doped phosphate-silica waveguide cores confined in silica cladding, with erbium homogeneously distributed within the core. See Fig. 3. The model is developed from an experimentally verified and accurate Er-doped fiber amplifier model [7] with changes made to fulfill the above-mentioned differences in structure. The circularly symmetric $L P_{01}$ mode 


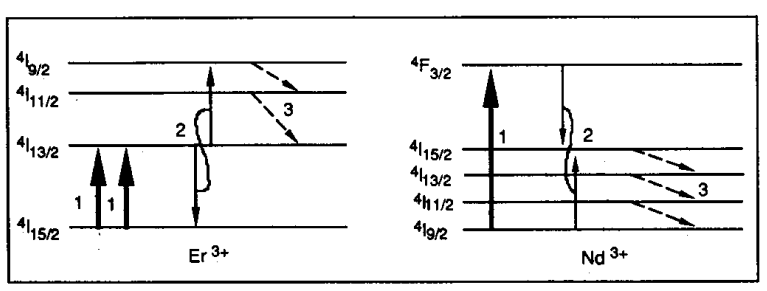

Fig. 2. Energy level diagram with the Russel-Saunders ${ }^{S} L_{J}$ terms, illustrating the upconversion process of $\mathrm{Er}$ ions (left) and the cross-relaxation process of $\mathrm{Nd}$ ions (right). The figures illustrate the evolution with time Thick arrow: photon absorption. Thin arrow: internal energy transfer. Dotted arrow: nonradiative transitions.

description has been replaced with the fundamental $E_{11}^{y}$ or $E_{11}^{x}$ modes, calculated by an accurate optimized effective index method [33]. This method is a variational method, where the trial function is the product of two slab waveguide solutions, $X\left(x, n_{x}\right)$ and $Y\left(y, n_{y}\right)$, one for the $x$ dependency and one for the $y$ dependency. The slab waveguides have core width $A$, height $B$, and core refractive indexes $n_{x}$ and $n_{y}$, respectively. $n_{x}$ and $n_{y}$ are chosen so the integral

$$
\int_{-\infty}^{\infty} \int_{-\infty}^{\infty} X Y\left[\frac{\partial^{2}}{\partial x^{2}}+\frac{\partial^{2}}{\partial y^{2}}+n^{2}(x, y) k^{2}\right] X Y d x d y
$$

is maximum. A term accounting for the passive waveguide loss is included in the propagation equations, and the dissipative ion-ion interactions are included through their influence upon the fluorescence lifetime. Since little information about quenching is available for highly concentrated Er-doped glasses, the empirical formula that relates the fluorescence lifetime $\tau_{f}$ to the ion concentration for Nd-doped glasses [9] is adapted, taking into account that only processes between excited ions occur for the Er system:

$$
\tau_{f}=\frac{\tau_{0}}{1+(x(x, y, z) \cdot \rho / Q)^{2}} .
$$

Here, $x(x, y, z)$ is the local population inversion, $\rho$ the $\mathrm{Er}$ concentration, $\tau_{0}$ the fluorescence lifetime in the limit where the concentration approaches zero, and $Q$ the quenching concentration where $\tau_{f}=\left(\frac{1}{2}\right) \tau_{0}$. The fluorescence lifetime combines all transitions from the metastable state through the relation $\tau_{f}=\left(\tau_{r}^{-1}+\tau_{n r}^{-1}\right)^{-1}$, where $\tau_{r}$ is the radiative and $\tau_{n r}$ the nonradiative lifetime. By inserting (1) in the rate equations, a cubic equation of the population inversion arises:

$$
\begin{aligned}
x(x, y, z)^{3}+ & {\left[\left(R_{P A}(x, y, z)+W_{S A}(x, y, z)\right.\right.} \\
+ & \left.\left.R_{P E}(x, y, z)+W_{S E}(x, y, z)\right) \tau_{0}+1\right] \\
& \cdot\left(\frac{Q}{\rho}\right)^{2} x(x, y, z) \\
- & \left(R_{P A}(x, y, z)+W_{S A}(x, y, z)\right)\left(\frac{Q}{\rho}\right)^{2} \tau_{0}=0 .
\end{aligned}
$$

$R_{P A}$ and $R_{P E}$ are the pump absorption and emission rates, respectively, and $W_{S A}$ and $W_{S E}$ are the signal absorption and emission rates [7]. The model takes into account the forwardand backward-amplified spontaneous emission, ASE, each presented in 150 frequency slots from 1.4 to $1.7 \mu \mathrm{m}$. The ASE,

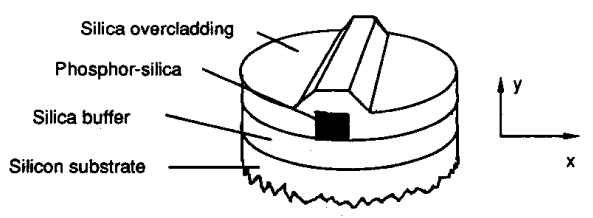

Fig. 3. Illustration of the considered silica-on-silicon component.

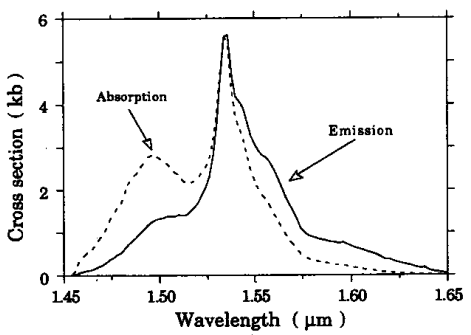

Fig. 4. Emission and absorption cross sections for erbium-doped P-silica waveguides ( $8 \mathrm{wt} \% \mathrm{P}_{2} \mathrm{O}_{5}$ ).

signal, and pump propagation yield 302 coupled differential equations that are solved by numerical integration through the waveguide. The emission cross section, shown in Fig. 4, was determined from a photoluminescence spectrum, measured on Er-doped P-glass [10] (8wt\% $\left.\mathrm{P}_{2} \mathrm{O}_{5}\right)$. The spectral shape is invariant to variations of the Er concentration. Absolute values of the emission cross section were found as described in [9], yielding the peak value of $\sigma_{e}(n)=5.6 \mathrm{~kb}$. The absorption cross section was calculated from the experimentally determined emission cross section using an approximate treatment of the McCumber theory [34]. This theory has shown the ability to provide accurate spectral values of cross sections for the ${ }^{4} I_{15 / 2} \leftrightarrow{ }^{4} I_{13 / 2}$ transition of Er-doped glasses and yields $\sigma_{a}(n)=5.6 \mathrm{~kb}$ at the peak. The absorption cross section at $0.98 \mu \mathrm{m}, \sigma_{a}\left(n_{p}\right)=1.3 \mathrm{~kb}$ was determined from a $0.7 \mathrm{~dB} / \mathrm{cm}$ pure optical absorption measurement [35] in an Er-doped P-glass channel waveguide.

\section{MODEL VERIFICATION}

Verification of the model was made by comparing calculations and measurements on a well-examined waveguide amplifier [35], having a buried waveguide core of $A_{m} \times$ $B_{m}=8 \times 7 \mu \mathrm{m}^{2}$, a length of $L_{m}=19.4 \mathrm{~cm}$, and a refractive index difference of $1.2 \%\left(8 \mathrm{wt} \% \mathrm{P}_{2} \mathrm{O}_{5}\right)$. A 0.980 $\mu \mathrm{m}$ pump was used, together with the $1.535 \mu \mathrm{m}$ signal in a copropagating configuration. The concentration-dependent background losses increased linearly from $\alpha_{p}=0.14$ to 0.28 $\mathrm{dB} / \mathrm{cm}$ for the pump, and from $\alpha_{s}=0.11$ to $0.22 \mathrm{~dB} / \mathrm{cm}$ for the signal for concentrations below $\rho=115 \cdot 10^{24} \mathrm{~m}^{-3}$. The pump power dependence upon the net gain was examined for a fixed mean $\mathrm{Er}$ concentration of $\rho_{m}=48 \cdot 10^{24} \mathrm{~m}^{-3}$. Calculations for different quenching concentrations above $Q=$ $17 \cdot 10^{24} \mathrm{~m}^{-3}$ are shown in Fig. 5 in comparison with the measurements (circles). $Q \rightarrow \infty$ corresponds to negligible effects of the ion-ion interactions, and yields a conflict of up to $10 \mathrm{~dB}$ with the measurements. Decreasing $Q$ diminishes this inconsistency, and $Q=17 \cdot 10^{24} \mathrm{~m}^{-3}$ yields a difference 


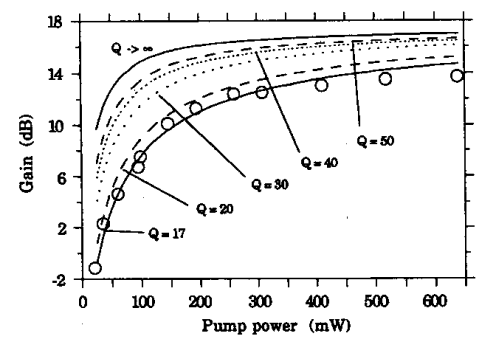

Fig. 5. Gain versus pump power, calculated for different $Q$ values (should be multiplied by $10^{24} \mathrm{~m}^{-3}$ ). Measurements (read from [12]) are shown as circles.

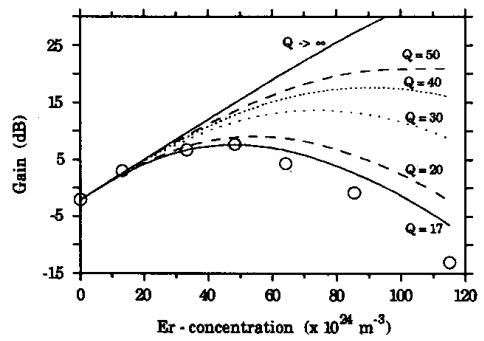

Fig. 6. Gain versus erbium concentration for different $Q$ values (should be multiplied by $10^{24} \mathrm{~m}^{-3}$ ). Measurements (read from [12]) are shown as circles.

of less than $1 \mathrm{~dB}$ in the entire pump power interval. The $\tau_{0}=13.7 \mathrm{~ms}$ lifetime reported in [10] was used in the calculations, but as different fabrication processes might yield different magnitudes of $\tau_{0}$, the correlation among gain, pump power, $\tau_{0}$, and $Q$ has been examined. Gain curves deviate only a few tenths of a decibel from the case with $\tau_{0}=13.7 \mathrm{~ms}$ if the $Q$ factor at the same time is increased $\left(Q=25 \cdot 10^{24} \mathrm{~m}^{-3}\right.$ for $\tau_{0}=8 \mathrm{~ms}$ ). In addition, significant $\mathrm{Er}$ ion-ion interactions have been observed at levels of $\sim 10^{24} \mathrm{~m}^{-3}$ for both pure silica and Ge-doped silica fibers [9], and a rapid fluorescence lifetime decrease reported at concentrations above $0.1 \mathrm{wt} \%$ $\left(8 \cdot 10^{24} \mathrm{~m}^{-3}\right)$ in the amplifier experiment [35].

The gain versus concentration dependence is examined in Fig. 6 for a fixed pump power of $100 \mathrm{~mW}$. Significant gain reductions are observed for concentrations above $10 \cdot 10^{24} \mathrm{~m}^{-3}$ (approximately ten times higher than the typical concentrations in Er-doped fibers). However, the pronounced gain differences to the case of no ion-ion interactions grow at higher concentrations to $50 \mathrm{~dB}$ at $\rho=115 \cdot 10^{24} \mathrm{~m}^{-3}$. By reducing the quenching concentration to $Q=17 \cdot 10^{24} \mathrm{~m}^{-3}$, the theoretical curve shows excellent agreement with the measured values for concentrations up to $60 \cdot 10^{24} \mathrm{~m}^{-3}$. Experimental data display a stronger deflection than the calculated in the remaining interval, perhaps due to even higher order nonlinear ion-ion interactions.

\section{RARE EARTH-DOPED COMPONENTS}

The present research within rare earth-doped integrated components can basically be grouped into three different categories: lasers, lossless $Y$ branches, and high-gain amplifiers, each group with optimal design parameters that deviate

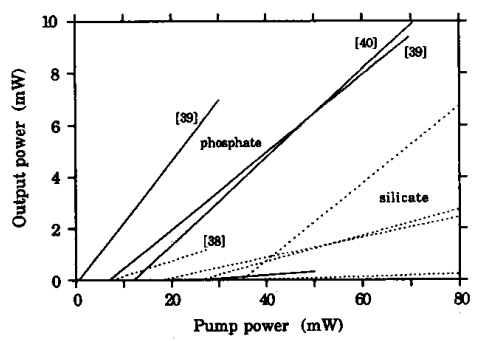

Fig. 7. Laser output power versus pump power for different integrated $\mathrm{Nd}$ lasers presented in the literature. Solid line: phosphate silica glass host. Dotted line: silicate glass host.

remarkably from the others. However, combinations of these, i.e., an amplifying $Y$ branch or a $Y$ branch laser with application for dual-output, mode-selected or $Q$-switched operation, may also find attraction.

Lasers are the most simple of these to realize, as they require relatively low rare earth concentrations. As in the case of active fiber development, the focus was first put on the transitions in $\mathrm{Nd}^{3+}$ because neodymium acts as a four-level laser system, and it is therefore easier to handle (achieve population inversion) than the three-level erbium system, plagued by ground state absorption. Although Nddoped glass waveguides were reported 20 years ago [36], the recent development was started by the presentation of an $\mathrm{Ag}^{+} \mathrm{K}^{+}$ion-exchanged waveguide in highly $\mathrm{Nd}$-doped silicate glass by Babukova et al. in 1985 [37]. But six years later in 1991, Mwarania et al. [38] presented the first truly singlemode glass channel waveguide laser. A $7.5 \mathrm{~mW}$ threshold and a $6 \%$ slope efficiency were measured on a $17 \mathrm{~mm}$ long $\mathrm{Na}^{+} \mathrm{K}^{+}$ion-exchanged device. A detailed review of this sixyear development is given in [5]. A pump threshold as low as $0.6 \mathrm{~mW}$ followed by a slope efficiency of $24 \%$ in a $5 \mathrm{~mm}$ long $\mathrm{Ag}^{+}$ion-exchanged waveguide [39] is, to the best of our knowledge, the most pronounced low-power Nd laser results obtained so far. The development of low-power Nd lasers is illustrated in Fig. 7. As can be seen, the most significant results are obtained with phosphate glasses (solid lines). Apart from the dominant $1.06 \mu \mathrm{m}$ transition [14] lasing, although less significant, others also have been reported around $0.91 \mu \mathrm{m}$ [40], $1.33 \mu \mathrm{m}$, and $1.36 \mu \mathrm{m}$ [8] for the Nd system.

Reports on erbium-doped glass lasers were seen in. 1991 with the presentation of an FHD-processed silica-on-silicon laser by Kitagawa et al. [12]. Co-doping with ytterbium is generally used with the Er system so as to enhance the absorption efficiency by utilizing the $\mathrm{Yb}\left({ }^{2} F_{5 / 2}\right)->\operatorname{Er}\left({ }^{4} I_{11 / 2}\right)$ energy transfer process [7]. To our knowledge, the best low-power Er-laser results obtained so far are a $16 \mathrm{~mW}$ threshold and a $10 \%$ slope efficiency from a $2.5 \mathrm{~mm}$ thick and $4 \mathrm{~mm}$ diameter $\mathrm{Er} / \mathrm{Yb}$-phosphate disk [30].

Lossless $Y$ branches can be viewed as a first step towards complex multichannel devices, as the $1 \times 2$ geometry can be extended to a large number of output ports. The lossless $Y$ branch requires an amplification high enough to compensate for the $3 \mathrm{~dB}$ splitting loss, the background loss, and possibly coupling loss; in all, about 5-7 dB of amplification is needed. $85 \mathrm{~mW}$ of $807 \mathrm{~nm}$ pump was used to overcome the splitting 


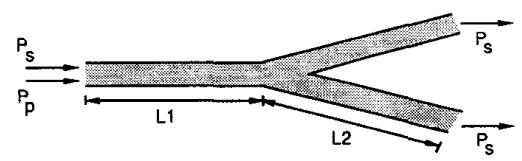

Fig. 8. $Y$ branch. Hatched area illustrates RE-doped area. $P_{s}$ : signal power. $P_{p}:$ pump power.

loss in a $24 \mathrm{~mm}$ long $\mathrm{Nd}$-doped device [41], defined by ion exchange in a silicate glass, while $60 \mathrm{~mW}$ of $980 \mathrm{~nm}$ pump was found sufficient to compensate for splitting and excess loss in a $23 \mathrm{~cm}$ Er-doped silica-on-silicon device [31]. In addition to the fabrication of single-output port amplifiers, the branch proportional $L_{1} /\left(L_{1}+L_{2}\right)$ is an extra design parameter that must be optimized to reduce the pump power required. $L_{1}$ and $L_{2}$ are the length of doped waveguide, respectively before and after the ramification, as illustrated in Fig. 8. Kitagawa [31] has reported the optimum position to be 0.9 for an Er-doped silica-on-silicon component.

Two significant deviating parameters for the high-gain amplifier are the rare earth concentration and the waveguide length, both considerably larger than for the laser and $Y-$ branch components. The promising silica-on-silicon components especially require very long lengths, but $170 \mathrm{~cm}$ long passive channel waveguides in a coiled configuration (bending radius of $5 \mathrm{~mm}$ ) have been realized [42] with low propagation loss $(0.038 \mathrm{~dB} / \mathrm{cm})$. Optimal design of this type of amplifier will be examined in detail below; here, it should be mentioned that the highest net gain reported for integrated glass amplifiers is $23 \mathrm{~dB}$ [31] from a $36 \mathrm{~cm}$ long erbium-doped $P$ silica-onsilicon component, pumped with $240 \mathrm{~mW}$ of $0.98 \mu \mathrm{m}$ light. Also, $10 \mathrm{~dB}$ net gain from an only $4.7 \mathrm{~mm}$ long $\mathrm{Er} / \mathrm{Yb}$-doped composite glass waveguide amplifier $(\sim 21 \mathrm{~dB} / \mathrm{cm})$ has been reported, applying only $30 \mathrm{~mW}$ of pump power [21].

\section{HIGH-GAIN AMPLIFIER OPTIMIZATION}

We will now select the high-gain component for optimization, and take as reference the above-reported amplifier [35] $\left(A_{m}, B_{m}, L_{m}, \rho_{m}\right)=(8 \mu \mathrm{m}, 7 \mu \mathrm{m}, 19.4 \mathrm{~cm}, 48$. $10^{24} \mathrm{~m}^{-3}$ ) applying $100 \mathrm{~mW}$ of pump power. Five different design parameters are evaluated, that is, waveguide length, core cross section, erbium concentration, pump power, and background loss. The reference gain, measured (symbols) and calculated (lower solid line), are shown again versus erbium concentration in Fig. 9, all determined from the fixed length of $19.4 \mathrm{~cm}$. An optimum length exists, however, for each single concentration, whereas the gain reaches its maximum. Shorter lengths yield an incomplete utilization of the pump, whereas the pump for longer lengths cannot invert the Er ions sufficiently. The length-optimized gain and the optimum length for the reference component are shown as dashed lines in Figs. 9 and 10 , respectively.

It is seen that the reference length (horizontal line) is less than half the optimum, and that the highest reference gain can be increased with $3 \mathrm{~dB}$ simply by changing the concentration to $\rho=30 \cdot 10^{24} \mathrm{~m}^{-3}$ and the length to $45 \mathrm{~cm}$. The evolution in the optimum waveguide length is seen to increase from a few $\mathrm{cm}$ at very high concentrations to $60 \mathrm{~cm}$ at $\rho=13 \cdot 10^{24} \mathrm{~m}^{-3}$.

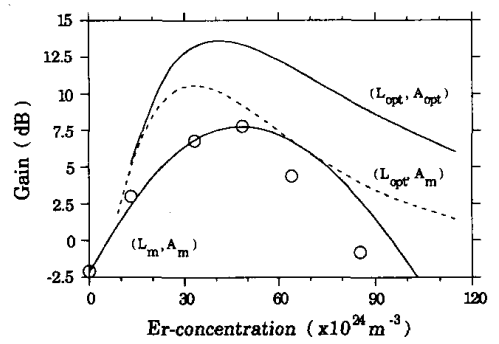

Fig. 9. Gain versus erbium concentration. Circles: measurements read from [35]. Lower solid line: reference calculation. Dotted line: length-optimized gain. Upper solid line: length- and core-optimized gain.

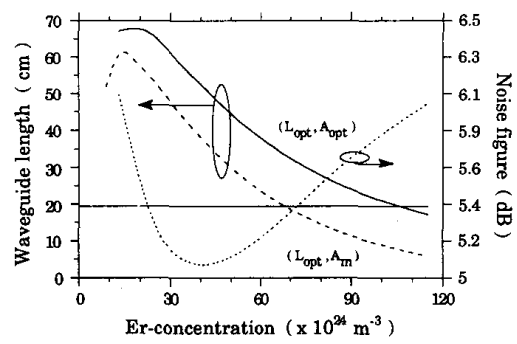

Fig. 10. Gain versus erbium concentration. Horizontal line: reference calculation. Dashed line: length-optimized gain. Upper solid line: length- and core-optimized gain. Dotted line: corresponding noise figure.

For smaller concentrations, the optimum length approaches zero because the total background loss becomes comparable with the gain accumulation. The length- and concentrationoptimized amplifier gain may be further increased if the waveguide core cross section is also optimized. By decreasing the reference core, the overlap integral between the Er-doped region and the mode profile is increased, followed by gain improvements. However, for very small cross sections, yielding long optimum lengths the accumulated background loss will diminish the gain seriously, so obviously an optimum exists. The upper solid curves in Figs. 9 and 10 represents calculations where the core cross section and the waveguide length have been optimized to maximum gain simultaneously for each concentration considered. A quadratic core cross section is considered to achieve a fundamental mode that matches the circularly symmetric fundamental mode of optical fibers. The core size optimization yields an additional $3 \mathrm{~dB}$ gain at the optimum concentration $\rho=41 \cdot 10^{24} \mathrm{~m}^{-3}$, a core width of $4.6 \mu \mathrm{m}$, and a length of $53 \mathrm{~cm}$. The optimum core size varies inversely proportional to the concentration from $6.2\left(\rho=10 \cdot 10^{24} \mathrm{~m}^{-3}\right)$ to $3.4 \mu \mathrm{m}\left(\rho=115 \cdot 10^{24} \mathrm{~m}^{-3}\right)$. Also, the evolution of the noise figure is shown for the latter optimization, yielding a minimum of $5.1 \mathrm{~dB}$. An even lower noise figure can be obtained with increasing gain by increasing the pump power, or for a fixed pump power (and decreasing gain), by reducing the waveguide length.

The above-determined design combination of $A, \rho$, and $L$ is optimum for a fixed pump power of $100 \mathrm{~mW}$. By changing the power, the design must be changed, which is why a threedimensional simultaneous optimization of these parameters is made for the pump power evolution. This is illustrated in Fig. 


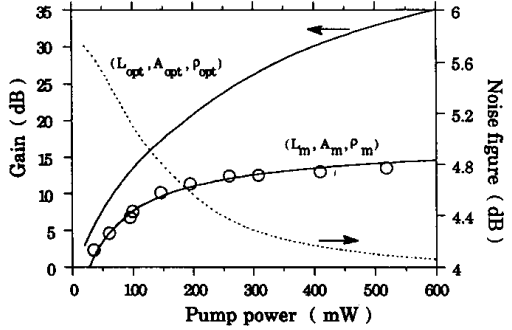

Fig. 11. Gain versus pump power. Circles: measurements read from [35] Lower solid line: reference calculation. Upper solid line: simultaneous length, core, and concentration optimization. Dotted line: corresponding noise figure.

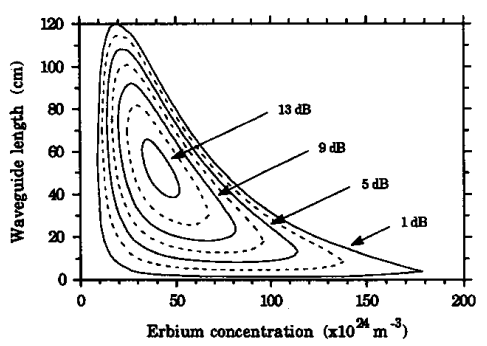

Fig. 12. Gain contour curves for different waveguide lengths and erbium concentrations, corresponding to the reference setup.

11 in comparison with the reference results. The resulting gain curve (upper solid line) clearly illustrates how a high-gain component can be obtained by proper design. $\left(\rho_{\mathrm{opt}}, A_{\mathrm{opt}}\right.$, $\left.L_{\text {opt }}\right)$ increase with pump power from $\left(31 \cdot 10^{24} \mathrm{~m}^{-3}, 4.1\right.$ $\mu \mathrm{m}, 29 \mathrm{~cm})$ at $20 \mathrm{~mW}$ of pump to $\left(61 \cdot 10^{24} \mathrm{~m}^{-3}, 5.4 \mu \mathrm{m}, 61\right.$ $\mathrm{cm}$ ) at $640 \mathrm{~mW}$. Gains above $35 \mathrm{~dB}$ are obtained in the latter situation, and the corresponding noise figure approaches $4 \mathrm{~dB}$.

The latter of the five design parameters to be examined is the background loss. To evaluate its influence upon the optimal amplifier design, we consider the gain contour curves for the reference amplifier, as shown in Fig. 12. This shows combinations of waveguides length and erbium concentration required to obtain a certain gain. Note that the shortest allowable length for a $13 \mathrm{~dB}$ amplifier with reference design is $38 \mathrm{~cm}$, while the shortest allowable concentration is $32 \cdot 10^{24}$ $\mathrm{m}^{-3}$. Consider, now, background losses that are concentration independent, $\alpha_{p}=\alpha_{s}=0.11 \mathrm{~dB} / \mathrm{cm}$ and $\alpha_{p}=\alpha_{s}=0.08$ $\mathrm{dB} / \mathrm{cm}$. This change has an enormous effect upon the design, and the expansion of the $5 \mathrm{~dB}$ (dotted) and $13 \mathrm{~dB}$ (solid lines) gain contour curves are shown in Fig. 13. The shortest lengths required for a $13 \mathrm{~dB}$ amplifier are halved to $22 \mathrm{~cm}(\alpha=0.11$ $\mathrm{dB} / \mathrm{cm})$ and $18 \mathrm{~cm}(\alpha=0.08 \mathrm{~dB} / \mathrm{cm})$. Minimum required concentrations are reduced to $21 \cdot 10^{24} \mathrm{~m}^{-3}(\alpha=0.11 \mathrm{~dB} / \mathrm{cm})$ and $14 \cdot 10^{24} \mathrm{~m}^{-3}$, respectively, while at the same time, the highest possible gain is increased to $31.8 \mathrm{~dB}$, all for a fixed pump power of $100 \mathrm{~mW}$.

The relationship among background loss, pump power, optimal gain, and erbium concentrations is clarified in Fig. 14 for a fixed waveguide width of $4.65 \mu \mathrm{m}$. In the situation with extremely small background loss, gains in excess of $40 \mathrm{~dB}$ can be reached with $60 \mathrm{~mW}$ of pump. The design is very critical to variations of $\alpha$, i.e., the slope around a state-of-the-art, realistic

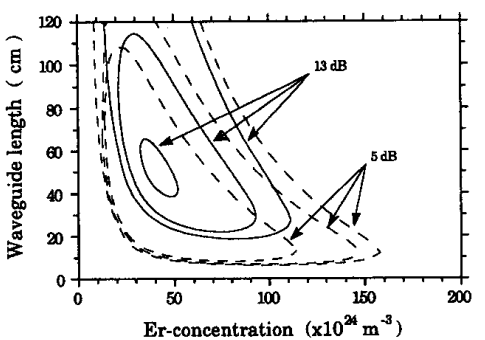

Fig. 13. 5-dB (dotted lines) and 13-dB (solid lines) gain contour curves for different waveguide lengths and erbium concentrations. Smallest curves with background losses as for the reference. Middle curves with $\alpha_{p}=\alpha_{s}=0.11$ $\mathrm{dB} / \mathrm{cm}$. Largest curves with $\alpha_{p}=\alpha_{s}=0.08 \mathrm{~dB} / \mathrm{cm}$.

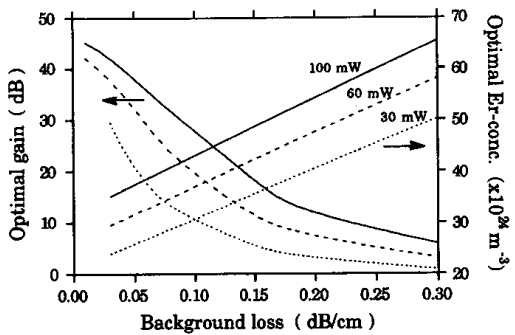

Fig. 14. Optimal gain and erbium concentration versus background loss for different pump power levels. $A=4.65 \mu \mathrm{m}$.

processed reproducible background loss of $0.08 \mathrm{~dB} / \mathrm{cm}$ is up to $300 \mathrm{~dB} /(\mathrm{dB} / \mathrm{cm})$. An almost linear relationship between background loss and optimal erbium concentration is observed for $\alpha>0.05 \mathrm{~dB} / \mathrm{cm}$. The slope of around $105 \mathrm{~m}^{-3} /(\mathrm{dB} / \mathrm{cm})$ for pump levels considered is slightly increasing versus pump power from $100 \mathrm{~m}^{-3} /(\mathrm{dB} / \mathrm{cm})\left(P_{p}=30 \mathrm{~mW}\right)$ to 130 $\mathrm{m}^{-3} /(\mathrm{dB} / \mathrm{cm})\left(P_{p}=500 \mathrm{~mW}\right)$. A somewhat more complicated evolution is seen from Fig. 15 to exist for the optimal waveguide length. High background losses $(>0.07$ $\mathrm{dB} / \mathrm{cm}$ ) yield increasing lengths versus pump power up to 100 $\mathrm{mW}$. However, for lower loss values, a maximum length is observed. This maximum length arises from the correlation and weighting between, on the one hand, the gain degradation caused by concentration increase and, on the other hand, gain degradation caused by length increase. It is remarkable that the optimum length is unchanged over a $50 \mathrm{~mW}$ pump power interval for $\alpha=0.07 \mathrm{~dB} / \mathrm{cm}$.

\section{PROCESS REPRODUCIBILITY}

Reproducibility of the design parameters during the different processingsteps will determine how accurately the aboveshown design curves can be followed. These process parameters are core width, length, and height, the refractive index difference, background loss, and erbium concentration. It is expected that a specified concentration can be met within $\pm 5 \%$ in the more controllable processes, although it might be much more uncertain for others. The refractive index and layer thickness are parameters related to the deposition process, and are strongly dependent upon deposition method and speed. A standard process yields typical variations over one 4 in 


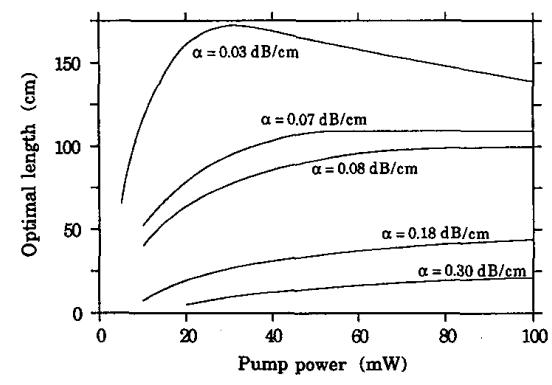

Fig. 15. Optimal waveguide length versus pump power for different back ground loss values. $A=4.65 \mu \mathrm{m}$.

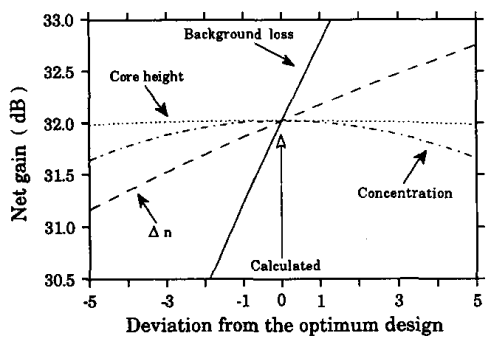

Fig. 16. Gain for different process parameter deviations from the optimum design. Five $x$-axis units correspond to a $5 \%$ thickness increase, $0.07 \% \Delta n$ increase, $5 \%$ increase in $\pi$, and $0.015 \mathrm{~dB} / \mathrm{cm}$ decreased background loss.

silicon wafer less than $\pm 0.007 \%$ in index for the core and $\pm 0.014 \%$ for the buffer, each layer with a variation of $\pm 3.0 \%$ in thickness. A wafer-to-wafer examination for up to 30 wafers in one deposition run [43] showed that $90 \%$ of all wafers produced had a refractive index difference, $\Delta n$ within $\pm 0.07 \%$ and a thickness within $\pm 5 \%$. The background loss is primarily determined by the etching step in the waveguide fabrication process. From an examination of 56 channel waveguides over two wafers, it is seen [43] that $2 / 3$ of all nondestroyed waveguides had a background loss variation of $\pm 0.015 \mathrm{~dB} / \mathrm{cm}$. An even lower standard deviation of $0.003 \mathrm{~dB} / \mathrm{cm}$ was recently reported among 19 channel waveguides $170 \mathrm{~cm}$ long on $\mathrm{Si}$ [42], although all were fabricated on the same wafer.

Consider the situation with $\alpha=0.08 \mathrm{~dB} / \mathrm{cm}$ as illustrated in Fig. 13, yielding a maximal gain of $31.8 \mathrm{~dB}$. If, also, the core cross section were optimized, the overall optimum design would be $(A, \rho, L)=\left(4.16 \mu \mathrm{m}, 43.7 \cdot 10^{24} \mathrm{~m}^{-3}, 102 \mathrm{~cm}\right)$, yielding the overall optimum gain of $32.0 \mathrm{~dB}$. Gain variations due to parameter deviations from this design are shown in Fig. 16 . The " 0 " point on the $x$ axis corresponds to the calculated design. Five units on the $x$ axis correspond to a $5 \%$ increase in core layer thickness, a $0.07 \%$ increase in refractive index difference, $5 \%$ increase in erbium concentration, and a 0.015 $\mathrm{dB} / \mathrm{cm}$ decrease in background loss. The variation of buffer layer thickness will have no effect on the performance as long as the buffer is sufficiently thick to avoid leakage to the substrate. Length and width of the channel waveguide are determined accurately by a lithographic process, and are therefore not considered to increase the uncertainties further.

As can be seen from Fig. 16, the background loss is the most critical parameter for the design. Due to the extremely long waveguide, the considered $0.015 \mathrm{~dB} / \mathrm{cm}$ increase will result in no less than a $4.2 \mathrm{~dB}$ gain decrease. If each process parameter were allowed to yield an absolute variation of $0.5 \mathrm{~dB}$ in gain among the realized waveguides, there would be no problems for concentration and thickness variations of $\pm 5 \%$. However, variations of $\Delta n$ should be less than $\pm 0.02 \%$ and the requirement to background loss would be less than $\pm 0.001 \mathrm{~dB} / \mathrm{cm}$, the latter being impossible. When design-optimized active fibers are fabricated, deviation from the specified performance, due to variations in the process parameters, may be corrected by adjusting the fiber length, while testing the realized component. This possibility of correction is different for active wafers. Once realized, the length cannot be adjusted, which is why, instead, a proper design should use a pump power level on the dynamic part of the gain-to-pump power curve so as to use the pump power for the last adjustment after the processing. It should, however, be noticed that the requirements for the background loss reduction remarkably drop with the wanted gain and thereby length of the waveguide.

\section{CONCLUSION}

A comprehensive model of an integrated, highly erbiumdoped phosphate glass amplifier has been found to reproduce measured gains up to concentrations of $60 \cdot 10^{24} \mathrm{~m}^{-3}$. The emission and absorption cross section peaks at $1.55 \mu \mathrm{m}$ of Er-doped phosphate silica are found to be $5.6 \cdot 10^{-25} \mathrm{~m}^{2}$. Ion-ion interactions are found to reduce the amplifier gain in excess of $50 \mathrm{~dB}$ for very high doping levels, and optimal erbium concentrations have been determined as approximately 30 to60 $\cdot 10^{24} \mathrm{~m}^{-3}$ for realistic background losses and pump power levels below $100 \mathrm{~mW}$. The optimal concentrations are found to increase linearly with a background loss of around $105 \mathrm{~m}^{-3} /(\mathrm{dB} / \mathrm{cm})$, having a slightly increasing slope for increasing pump power levels. The optimal active waveguide length is found to be up to $175 \mathrm{~cm}$ for background losses of $0.03 \mathrm{~dB} / \mathrm{cm}$, and strongly decreases for increasing loss. Net gains in excess of $40 \mathrm{~dB}$ are predicted for only $60 \mathrm{~mW}$ of pump power in the situation with small background loss. Performance degradation due to reported variations in process parameters during a multiple wafer fabrication are found to be very significant. The background loss is the most critical parameter to control; a deviation of $0.015 \mathrm{~dB} / \mathrm{cm}$ from the assumed value in the design results in a $4.2 \mathrm{~dB}$ gain decrease. Variations below $0.5 \mathrm{~dB}$ require control of core thickness better than $\pm 5 \%, \Delta n$ better than $\pm 0.02 \%$, and background losses better than $\pm 0.001 \mathrm{~dB} / \mathrm{cm}$. The latter requirement diminishes significantly with the wanted amplifier gain, and thereby required length.

\section{ACKNOWLEDGMENT}

The authors thank Dr. E. Nicolaisen for fruitful discussions.

\section{REFERENCES}

[1] P. Laporta, S. De Silvestri, V. Magni, and O. Svelto, "Diode-pumped cw bulk Er:Yb:glass laser," Opt. Lett., vol. 16, no. 24, pp. 1952-1954, 1991. 
[2] P. Laporta, S. Longhi, S. Taccheo, and O. Svelto, "Single-mode cw erbium-ytterbium glass laser at $1.5 \mu \mathrm{m}$," Opt. Lett., vol. 18 , no. 1, pp. 31-33, 1993.

[3] J.A. Hutchinson and T. H. Allik, "Diode array-pumped Er, Yb: Phosphate glass laser," Appl. Phys. Lett., vol. 60, no. 12, pp. 1424-1426, 1992.

[4] N. A. Sanford, K. J. Malone, and D. R. Larson, "Integrated-optic waveguide glass lasers," in Proc. Opt. Fiber Commun., OFC'91, San Diego, CA, 1991, paper TuG2, p. 27.

[5] E. Lallier, "Rare earth-doped glass and $\mathrm{LiNbO}_{3}$ waveguide lasers and optical amplifiers," Appl. Opt., vol. 31, no. 25, pp. 5276-5282, 1992.

[6] S. Iraj Najafi, Ed., Introduction to Glass Integrated Optics. Boston, London: Artech House, 1992.

[7] A. Bjarkelv, Optical Fiber Amplifiers: Design and System Applications. Boston, London: Artech House, 1993.

[8] H. Aoki, O. Maruyama, and Y. Asahara, "Glass waveguide laser operated around $1.3 \mu \mathrm{m}$," IEE Electron. Lett., vol. 26, no. 22, pp. 1910-1911, 1990.

[9] W. J. Miniscalco, "Erbium-doped glasses for fiber amplifiers at 1500 nm," J. Lightwave Technol, vol. 9, no. 2, pp. 234-250, 1991.

[10] A. Polman, D. C. Jacobson, D. J. Eaglesham, R. C. Kistler, and J. M. Poate, "Optical doping of waveguide materials by $\mathrm{MeV} \mathrm{Er}$ implantation," J. Appl. Phys., vol. 70, no. 7, pp. 3778-3784, 1991.

[11] K. Shuto, K. Hattori, T. Kitagawa, Y. Ohmori, and M. Horiguchi, "Erbium-doped phosphosilicate glass waveguide amplifier fabricated by PECVD," IEE Electron. Lett., vol. 29, no. 2, pp. 139-141, 1993.

[12] T. Kitagawa, K. Hattori, M. Shimizi, Y. Ohmori, and M. Kobayashi, "Guided-wave laser based on erbium-doped silica planar lightwave circuit," IEE Electron. Lett., vol. 27, no. 4, pp. 334-335, 1991.

[13] J. A. Bebbington, G. Barbarossa, J. R. Bonar, and J. S. Aitchison, "Rare earth doped silica waveguides on $\mathrm{Si}$ fabricated by flame hydrolysis deposition and aerosol doping," Appl. Phys. Lett., vol. 62, no. 4, pp. 337-339, 1993.

[14] R. Tumminelli, F. Hakimi, and J. Haavisto, "Integrated-optic Nd:glass laser fabricated by flame hydrolysis deposition using chelates," Opt. Lett., vol. 16, no. 14, pp. 1098-1100, 1991.

[15] J. Shmulovich, Y. H. Wong, G. Nykolak, P. C. Becker, R. Adar, A J. Bruce, D. J. Meuhlner, G. Adams, and M. Fishteyn, " $15 \mathrm{~dB}$ net gain demonstration in $\mathrm{Er}^{3+}$ glass waveguide amplifiers on silicon," in Proc. Conf. Opt. Fiber Commun., OFC'93, San Jose, CA, vol. III, Feb. 1993, postdeadline paper PD18, pp. 75-78.

[16] M. Nakazawa and Y. Kimura, "Electron-beam vapour-deposited erbiumdoped glass waveguide laser at $1.53 \mu \mathrm{m}$," IEE Electron. Lett., vol. 28 , no. 22, pp. 2054-2056, 1992.

[17] E. F. Schubert, A. M. Vredenberg, N. E. J. Hunt, Y. H. Wong, P. C. Becker, J. M. Poate, D. C. Jacobsen, L. C. Feldman, and G. J. Zydzik, "Giant enhancement of luminiscence intensity in Er-doped $\mathrm{Si} / \mathrm{SiO}_{2}$ resonant cavities," Appl. Phys. Lett., vol. 61, no. 12, pp. 1381-1383, 1992.

[18] S. I. Najafi, W. Wang, J. F. Currie, R. Leonelli, and J. L. Brebner, "Fabrication and characterization of neodymium-doped glass waveguides," IEEE Photon. Technol. Lett., vol. 1, no. 5, pp. 109-110, 1989.

[19] E. Snoeks, G. N. van den Hoven, and A. Polman, "Optical doping of soda-lime-silicate glass with erbium by ion implantation," J. Appl. Phys., vol. 73 , no. 12 , pp. $8179-8183,1993$.

[20] X. H. Zheng and R. J. Mears, "Planar optical waveguides formed by erbium ion exchange in glass," Appl. Phys. Lett., vol. 62, no. 8, pp. 793-795, 1993.

[21] W. J. Wang, S. I. Najafi, S. Honkanen, Q. He, C. Wu, and J. Glinski, "Erbium-doped composite glass waveguide amplifier," IEE Electron. Lett., vol. 28, no. 20, pp. 1872-1873, 1992.

[22] B. Pálsdóttir and C. Larsen, "Short erbium doped fiber amplifiers optimized for high output power," in Proc. European Conf. Opt. Commun. ECOC'93, Montreux, Switzerland, Sept. 1993, paper Tu4, pp. 181-184.

[23] F. Ladouceur, J. D. Love, and T. J. Senden, "Measurement of surface roughness in buried channel waveguides," IEE Electron. Lett., vol. 28 , no. 14, pp. 1321-1322, 1992.

[24] R. Adar, Y. Shani, G. H. Henry, R. C. Kistler, G. E. Blonder, and N. A Olsson, "Very low loss phosphorus-doped silica-on-silicon waveguides measured using a ring resonator," in Proc. Conf. Opt. Fiber Commun., OFC'91, San Diego, CA, Feb. 1991, paper TuF5, p. 22.

[25] S. Helmfrid and G. Arvidsson, "Numerical calculation of the small signal gain in Er:Ti:LiNbO3 channel waveguides," IEEE Photon. Technol. Lett., vol. 3, no.7, pp. 635-637, 1991.

[26] T. Rasmussen, A. Bjarklev, J. H. Povlsen, O. Lumholt, and K. Rottwitt, "Numerical modeling of an integrated erbium-doped glass laser," Fiber and Integrated Opt., vol. 10, pp. 239-243, 1992

[27] W. Sohler, "Rare earth doped LiNbO3 waveguide amplifiers and lasers," in Waveguide Optoelectronics, J. H. Marsh and R. M. De la Rue, Eds. Norwell, MA: Kluwer Academic, 1992.

[28] O. Lumholt, T. Rasmussen, and A. Bjarklev, "Modelling of extremely high concentration erbium-doped silica waveguides," IEE Electron. Lett., vol. 29, no. 5, pp. 495-496, 1993.

[29] M. Federighi, I. Massarek, and P. F. Trwoga, "Optical amplification in thin optical waveguides with high Er concentration," IEEE Photon Technol. Lett., vol. 5, no. 2, pp. 227-229, 1993.

[30] P. Laporta, S. Longhi, S. Taccheo, and O. Suelto, "Analysis and modelling of the erbium-ytterbium glass laser," Opt. Commun., vol. 100, no. $1,2,3,4$, pp. 311-321, 1993.

[31] T. Kitagawa, "Rare-earth-doped planar waveguide amplifiers," in Proc. OAA'93, Yokohama, Japan, 1993, paper MC1, pp. 136-139.

[32] P. Blixt, J. Nilsson, J. Babonas, and B. Jaskorzynska, "Excited-state absorption at $1.5 \mu \mathrm{m}$ in $\mathrm{Er}^{3+}$-doped fiber amplifiers," in Proc. Opt. Amplifiers and Their Appl. Topical Meeting, OAA'92, Sante Fe, NM, June 1992, paper WE2, pp. 63-66.

[33] T. Rasmussen, J. H. Povlsen, A. Bjarklev, O. Lumholt, B. Pedersen, and K. Rottwitt, "Detailed comparison of two approximate methods for the solution of the scalar wave equation for a rectangular optical waveguide," J. Lightwave Technol., vol. 11, no. 3, pp. 429-433, 1993.

[34] W. J. Miniscalco and R. S. Quimby, "General procedure for the analysis of $\mathrm{Er}^{3+}$ cross sections," Opt. Lett., vol. 16, no. 4, pp. 258-260, 1991.

[35] T. Kitagawa, K. Hattori, K. Shuto, M. Yasu, M. Kobayashi, and M. Horiguchi, "Amplification in erbium-doped silica-based planar lightwave circuits," in Proc. Opt. Amplifiers and Their Appl. Topical Meeting, $O A A^{\prime} 92$, Santa Fe, NM, June 1992, paper PD1.

[36] H. Yajima, S. Kawase, and Y. Sekimoto, "Amplification at $1.06 \mu \mathrm{m}$ using a Nd:glass thin-film waveguide," Appl. Phys. Lett., vol. 21, no. 9, pp. $407-409,1972$.

[37] M. V. Babukova, V. A. Verenberg, L. B. Glebov, N. V. Nikonorov G. T. Petrovskii, and V. S. Terpugov, "Investigation of neodymium silicate glass diffused waveguides," Sov. J. Quantum Electron., vol. 15, pp. 1304-1305, 1985 .

[38] E. K. Mwarania, L. Reekie, J. Wang, and J. S. Wilkinson, "Lowthreshold monomode ion-exchanged waveguide lasers in neodymiumdoped BK-7 glass," IEE Electron. Lett., vol. 26, no. 16, pp. 1317-1318, 1990.

[39] H. Aoki, HOYA Corp. R\&D Cen., private correspondence.

[40] K. J. Malone, N. A. Sanford, and J. S. Hayden, "Integrated optic laser emitting at 906, 1057 and $1358 \mathrm{~nm}$," IEE Electron. Lett., vol. 29, no. 8, pp. 691-693, 1993.

[41] N. A. Sanford, K. J. Malone, D. R. Larson, and R. K. Hickernell, "Ybranch waveguide glass laser and amplifier," Opt. Lett., vol. 16, no. 15, pp. $1168-1170,1991$

[42] Y. Ohmori, "Passive and active silica waveguides on silicon," in Proc. European Conf. Opt. Commun., ECOC'93, Montreux, Switzerland, Sept. 1993, paper MoP1, pp. 19-26.

[43] G. Grand, S. Valette, G. J. Cannell, J. Aarnio, and M. del Giudice, "Fibre pigtailed silicon based low cost passive optical components," in Proc European Conf. Opt. Commun., ECOC'90, 1990, pp. 525-528.

Ole Lumholt, photograph and biography not available at the time of publication.

Anders Bjarklev(M'92), photograph and biography not available at the time of publication.

Thomas Rasmussen, photograph and biography not available at the time of publication.

Christian Lester, photograph and biography not available at the time of publication. 